\title{
The repton model of gel electrophoresis
}

\author{
G. T. Barkema ${ }^{a}$ and M. E. J. Newman ${ }^{b}$ \\ ${ }^{a}$ Institute for Advanced Study, Olden Lane, Princeton, NJ 08540. U.S.A. \\ ${ }^{\mathrm{b}}$ Santa Fe Institute, 1399 Hyde Park Road, Santa Fe, NM 87501. U.S.A.
}

\begin{abstract}
We discuss the repton model of agarose gel electrophoresis of DNA. We review previous results, both analytic and numerical, as well as presenting a new numerical algorithm for the efficient simulation of the model, and suggesting a new approach to the model's analytic solution.
\end{abstract}

\section{Introduction}

Gel electrophoresis is a technique of great importance in the rapidly-growing fields of molecular genetics and genetic engineering. It provides a simple and economical way of measuring the lengths of polymer strands and, if necessary, refining them by length as well. In this article we will consider the physics of the agarose gel process, which is commonly applied to DNA, although it can in principle be applied to other polymers, such as RNA for example. (The other common variety of process is polyacrylamide gel electrophoresis, whose physics is somewhat different and not well modelled using the techniques described here.)

The basic idea is very simple. DNA or other polymer strands of a variety of unknown lengths are deposited in a thin layer of gel composed of agarose powder and a buffer solution which imparts a charge to each base pair or monomer. An electric field is applied horizontally and the charged DNA migrates under its influence. The rate of migration is found to depend on the length of the strands, with shorter strands travelling further in a given time than longer ones. After a period of some hours, the initial mixture of strands will have become separated and spread over several inches of the gel, allowing the experimenter to measure the relative concentrations of strands of different lengths, or cut particular areas out of the gel, thereby isolating strands with a particular range of lengths. 
Given the technological importance of the method, there is much interest in learning precisely what the mechanisms of gel electrophoresis are and how the migration rate depends on strand length, applied electric field, and the properties of the agarose gel. It is known that the agarose forms long strands in the gel which cross-link and impede the movement of the DNA. Furthermore, the pores between these strands have a size which is roughly equal to the persistence length of the DNA, with the result that the DNA is prevented from moving transversely to its length; its only mode of transport is a snakelike slithering along its length through the pores of the gel, a motion which de Gennes [1] has dubbed "reptation": movement of a polymer along its own length by diffusion of stored length. Many attempts have been made to model the dynamics of reptation, with varying degrees of sophistication, but interestingly one of the most quantitatively successful of these is also the simplest of the lot, the so-called "repton model". This model was first introduced by Rubinstein in 1987 [2] and has been extensively studied by various authors [38], including Widom and co-workers [9-11], who gave both analytic solutions of the model for short strand lengths and also extensive numerical simulations using a number of novel Monte Carlo techniques.

The outline of this paper is as follows. In Section 2 we describe the repton model and discuss its relationship to gel electrophoresis experiments. In Sections 3 and 4 we review some of the analytic and numerical results for the model. In Section 5 we introduce a mapping of the model to a hard-sphere particle model which has allowed us to perform more accurate numerical calculations and we present preliminary results from these calculations. We also discuss briefly how the particle mapping implies a connection between the repton model and the so-called asymmetric exclusion models, a link which may ultimately lead to an exact solution of the problem. In Section 6 we give our conclusions.

\section{The repton model}

The repton model of polymer reptation in a gel is illustrated in Figure 1. The gel is approximated by a collection of pores arranged on a square lattice. These are represented by the diagonal grid of squares in the figure. (The lattice is shown as two dimensional in Figure 1, although the real system is three dimensional. However, as discussed in Section 2.1, the properties of the model are independent of the number of dimensions of the lattice, so we may as well stick with two for clarity.) We represent the DNA or other polymer as a chain of $N$ polymer segments or "reptons", the black dots in the figure. (The reptons are not the same thing as base pairs; rather each one corresponds to one persistence length of DNA. Depending on the conditions of the experiment, the persistence length of DNA is between about 150 and 300 base pairs, as 
discussed in Section 2.2.) Reptons move from pore to adjacent pore diagonally according to the following rules:

(1) Reptons in the interior of the chain move only along the line of pores occupied by the chain. Thus a repton in the interior of the chain can only move to an adjacent pore if that pore is already occupied by one of its neighbouring reptons in the chain. Note that a consequence of this restriction is that if three or more neighbouring reptons in the chain find themselves all in one pore, only the two with connections to other reptons outside this pore are allowed to move.

(2) At least one repton must remain in each pore along the chain but otherwise the number of reptons in a pore is unrestricted. This means that a repton in the interior of the chain can only leave a pore if one of its neighbours is also in that pore and stays behind when it leaves. This second rule gives the polymer some elasticity without making it infinitely stretchy.

(3) The two reptons at the ends of the polymer chain can move to adjacent pores provided that rule (ii) is not violated.

In a real polymer there are additional excluded-volume effects due to the finite space occupied by the polymer, as well as a number of other physical processes which are not included in the repton model. It is assumed that these effects make only a small contribution to the behaviour of the polymer under electrophoresis.

Since we are interested in the rate at which DNA drifts through the gel, we also need to define the time-scale on which moves take place. To do this, we make the assumption that thermal fluctuations continually drive all the reptons to attempt moves to adjacent squares on the grid. We assume that every such move is as likely to be attempted as every other at any particular time, and we choose the time-scale such that each such move will be attempted once, on average, per unit time. (Not all of these moves will actually take place, since many of them will be rejected for violating one of the rules above. However, the moves which are allowed each take place with equal probability per unit time. There are no energies associated with the different states, and so no Boltzmann weights making one move more likely than another. The dynamics of the model is purely entropic.)

The model described so far is just a model of polymer diffusion. All the motions are random thermal ones and there is no applied electric field. To make a model of electrophoresis, we assign to each repton the same negative electric charge, mimicking the charging effect of the buffer solution in the experiment. Then we apply a uniform electric field to the model along the $x$-axis (the horizontal direction in the figure), breaking the spatial symmetry. As a result, instead of unit rates for the allowed moves in the positive and negative $x$-directions, the 

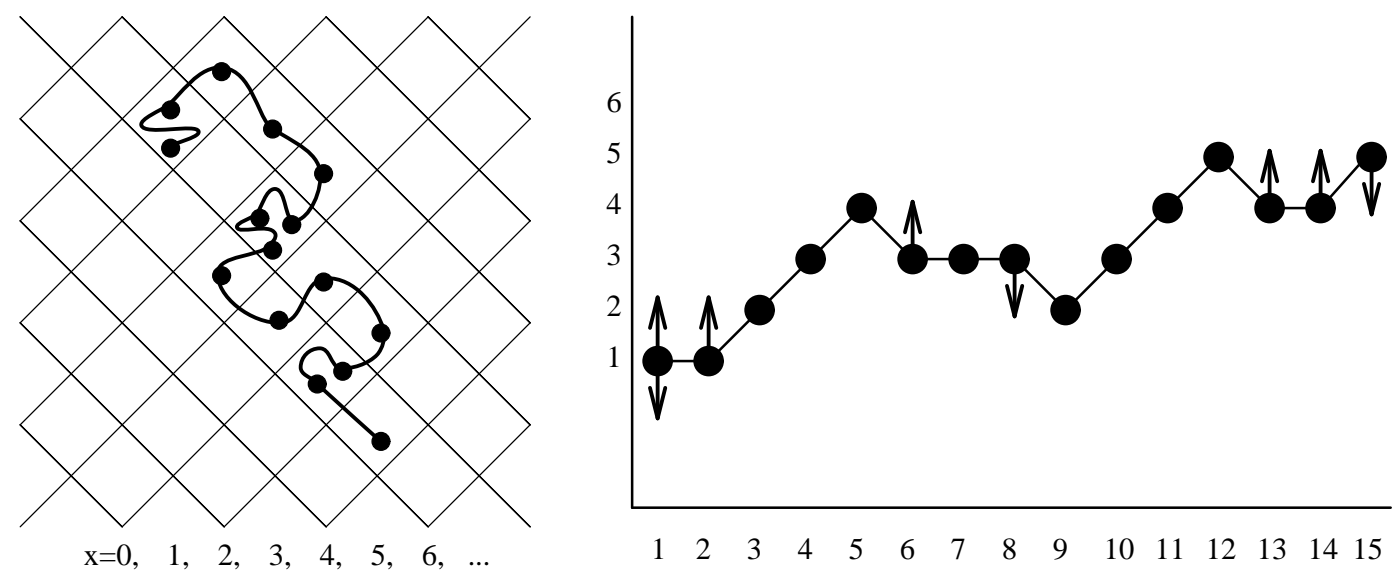

Fig. 1. A typical configuration of the repton model (left). If the reptons are numbered along the chain, starting with the end in the top-left corner, we can characterize this configuration by plotting the $x$-position as a function of the repton number. This is done in the right side of the figure. This is the projected model described in the text. Allowed moves are denoted by arrows.

rates become $\exp (-E / 2)$ in the positive direction and $\exp (E / 2)$ in the negative one, where $E$ is a new parameter which is proportional to the applied field (see Section 2.2). The resulting model describes the qualitative features of DNA electrophoresis surprisingly well, at least for longer strands of DNA (above about $1 \mathrm{~kb}$ ). Many details of the real system are missing, such as mechanical properties of the polymer, effects of counterions, the inhomogeneity of the gel, and the gel concentration, and to be truly realistic the model would have to include these features as well. However, we can extract a lot of useful information from the simple model we have already.

\subsection{The projected repton model}

The drawing in Figure 1 is two-dimensional, but real electrophoresis experiments are three-dimensional. Does this matter? As we mentioned briefly above, it does not, for the following reason. We are interested in calculating the drift velocity of the polymer for the case of a uniform electric field in the negative $x$-direction, which means we are interested only in the $x$-coordinates of the polymer; the values of the other coordinates are irrelevant. Thus, we might as well project the polymer onto the $x$-axis and ignore the other axes. We do precisely this on the right hand side of Figure 1, where we have numbered the reptons along the length of the chain and then plotted the $x$-coordinate as a function of repton number. This one-dimensional projection of the model proves very convenient both for analytic and numerical calculations, and we will mainly be discussing this form of the model from here on. 
Comparing the states of the projected model with those of the original higherdimensional version, we can easily work out how the dynamics looks after projection. First, reptons in the projected chain must either be at the same height as, or one place higher or lower than their neighbours, and each possible move consists of moving one repton either up or down one place. However, just satisfying these constraints is not enough. Consider, for instance, the repton numbered 7 in Figure 1. This repton is not allowed to move either up or down, even though the final configuration following such a move would be a perfectly legitimate one. This fact can easily be verified by going back to the higher-dimensional representation and examining repton 7 . In fact there is essentially only one type of move allowed in the projected model, in which a repton which has one of its neighbours on the level above or below it and one on the same level is allowed to move up or down respectively. The reader may like to verify that such moves are indeed the equivalent of reptation in the higher-dimensional version of the model. For the particular configuration shown in Figure 1, the allowed moves for the projected model are shown with arrows.

This projection of the repton model onto one dimension works just as well from three dimensions as from two, or indeed from any higher number of dimensions. The final rules for the dynamics of the projected model are just the same in each case, and so we conclude that the properties of the model are independent of the dimensions of the lattice on which it is built.

\subsection{Values of the parameters in the model}

Before we can investigate the properties of the repton model, we need to get an idea of the sort of values that the parameters $N$ and $E$ should take. The parameter $N$ measures the length of the polymer in multiples of the persistence length. Double-stranded DNA is quite stiff, and has a persistence length of around $150 \mathrm{bp}$, or about $400 \AA$. It can become stiffer still as a result of self-repulsion when it acquires an electric charge. Since the size of the charge picked up per base pair depends on the strength of the buffer solution used in the experiment, the actual persistence length varies from one experiment to another. In some cases it can be as high as $800 \AA$.

But $N$ also plays another role in the repton model. As we said above, the diagonal lattice of squares in Figure 1 represents the pores in the agarose gel, and the lattice spacing represents the typical pore size. But in this model, the maximum distance from one repton to the next is one lattice spacing, so $N$ is also effectively the length of the polymer in multiples of the pore size. Thus, the repton model will only be an effective model of DNA reptation if the persistence length and the pore size are roughly equal. The pore sizes in 
agarose gels have not been determined with great accuracy, but for the typical $1 \%$ agarose gel they are estimated to be in the range of 1000 to $3000 \AA$. Thus the two length scales are indeed of the same order of magnitude, and this goes some way towards explaining the success of the model. By contrast, the persistence length of RNA is only one or two bp or about 3 to $5 \AA$ since RNA is largely single-stranded. This length scale differs too greatly from the agarose pore size to allow the two scales to be well represented by the one parameter in the repton model, and as a result the model does not make good predictions about RNA electrophoresis experiments.

The dimensionless parameter $E$ is defined so as to be proportional to the electric field applied to the system. The quantity $\exp (E)$ is the ratio between the probability for a repton to move one step down the slope of the electric potential and the probability for moving one step up it, and so should be equal to the ratio of the Boltzmann factors for making those two moves. This means that

$$
E=\frac{\sqrt{2} a q E_{f}}{k T}
$$

where $a$ is the lattice parameter (i.e., the pore size), $q$ is the charge per repton (i.e., per persistence length), and $E_{f}$ is the applied electric field. The numerator here represents the energy needed to move a repton a distance $\sqrt{2} a$ (the $x$ distance between two nearest neighbours of the same lattice site) against the electric force $q E_{f}$ acting on it.

The charge $q$ is on the order of one electron charge $e$ per base pair, or about $150 \times 1.6 \times 10^{-19} \mathrm{C}$ per repton. Using typical values for the other parameters, we find that

$$
E \sim 10^{-3} E_{f}
$$

when $E_{f}$ is measured in $\mathrm{Vm}^{-1}$. This figure is bourne out by the experimental results of Barkema, Caron and Marko [12].

\section{$3 \quad$ Exact calculations}

In the limit of infinite length, Prähofer [13] has shown that the diffusion constant of the repton model is exactly equal to $1 /\left(3 N^{2}\right)$. However, the model shows strong finite size corrections for values of $N$ corresponding to the typical lengths of DNA strands used in experimental work, making it important that we calculate its behaviour for finite lengths also. For short chains one approach which has proved fruitful is the matrix technique employed by Widom 
and co-workers $[9,10]$. It is clear that for a strand of any finite length one can enumerate all possible states of the projected repton chain. Each link of the chain can be in one of three states - horizontal, or sloping in one of two directions. (There is an overall translation degree of freedom, vertically in Figure 1, but for the moment we are only concerned with the states of the chain itself.) For a chain of $N$ reptons, there are $3^{N-1}$ such states, and we can write down a transition rate matrix $\mathbf{T}(E)$, such that the element $T_{i j}$ is the probability that the chain will be in a state $j$ if it was in a state $i$ one move earlier. The off-diagonal elements take values proportional to $\mathrm{e}^{ \pm E / 2}$ if the states $i$ and $j$ differ by an allowed move of exactly one repton, and are zero otherwise. The diagonal ones are chosen to make the sum of the elements in each column equal to unity.

The probability $p_{i}$ of a particular state appearing in a large ensemble of identical chains, in the limit $t \rightarrow \infty$, is given by the $i^{\text {th }}$ element of the eigenvector corresponding to the slowest-decaying eigenmode of this matrix. Widom et al. [9] showed that these probabilities are related to the diffusion velocity $v$ of the chain via

$$
v=\frac{1}{N} \sum_{i}\left(\mathrm{e}^{E / 2} r_{i}-\mathrm{e}^{-E / 2} s_{i}\right) p_{i}
$$

where $r_{i}$ and $s_{i}$ are respectively the number of reptons which can lawfully move up and down when the chain is in state $i$. The diffusion constant $D$ of the polymer strand in the gel is given in terms of this velocity by the NernstEinstein relation:

$$
D=\lim _{E \rightarrow 0} \frac{v}{N E}
$$

for a system in which there is one dimensionless unit of charge on each repton. The only obstacle to the exact calculation of the diffusion constant then is the limit taken in Equation (4). Widom et al. circumvented this problem by noting that in the zero-field case the solution for the probabilities $p_{i}$ is simple -all states are equally probable and $p_{i}=3^{-(N-1)}$ for all $i$-and perturbing around this solution to find the small $E$ behaviour:

$$
p_{i}=\frac{1}{3^{N-1}}\left[1+\frac{1}{2} a_{i} E+\mathrm{O}\left(E^{2}\right)\right]
$$

Starting with the transition rate matrix, they showed that the coefficient $a_{i}$ is given by the solution of a set of linear equations

$$
\sum_{i \leftrightarrow j}\left(a_{i}-a_{j}\right)=-2\left(s_{i}-r_{i}\right),
$$


where the notation $i \leftrightarrow j$ means that the state $i$ can be reached from $j$ by one allowed move. Along with the subsidiary condition

$$
\sum_{i} a_{i}=0
$$

this set of equations determines the values of the coefficients $a_{i}$ for all states of the chain. Using Equation (5) we can then show that the diffusion constant is given in terms of these coefficients by

$$
D=\frac{1}{2 N^{2}}\left[\frac{4}{9}(N+4)-\frac{1}{3^{N-1}} \sum_{i}\left(s_{i}-r_{i}\right) a_{i}\right] .
$$

The only catch of course is that there are $3^{N-1}$ equations in (6), so that solving them involves the diagonalization of a $3^{N-1} \times 3^{N-1}$ matrix and, sparse though that matrix is, the problem rapidly grows beyond the capabilities of even the most powerful computers. Widom et al. computed the diffusion coefficients for systems with values of $N$ up to 5 . These results were extended to $N=12$ by Szleifer and Bisseling (unpublished) using a special purpose computer. However, given the exponential increase in the magnitude of the diagonalization problem with increasing $N$, it seems unlikely that this method will be taken any further.

\section{Simulation results}

In order to study chains of more than twelve reptons, we turn to Monte Carlo calculations. In their simplest form, these calculations are a direct implementation of the repton dynamics described in Section 2. In each step of the simulation we choose a repton at random from the chain and move it either in the direction of the electric field or against it, with probabilities proportional to $\mathrm{e}^{ \pm E / 2}$. In order that we attempt the correct number of moves per unit time, a move should correspond to a time interval of

$$
\Delta t=\frac{1}{N\left(\mathrm{e}^{E / 2}+\mathrm{e}^{-E / 2}\right)}=\frac{1}{2 N \cosh (E / 2)}
$$

We also need to make an estimate of the correlation time $\tau$ of the simulation, so that we know how many Monte Carlo steps we need to run for. For small electric fields the typical end-to-end distance of the chain is $\sqrt{N}$, and on average the chain reaches a new independent configuration every time it moves a length approximately equal to this distance. The correlation time is then 
given by Equation (4) thus:

$$
\tau=\frac{\sqrt{N}}{v}=\frac{\sqrt{N}}{D N E}=\frac{3 N^{3 / 2}}{E} .
$$

This estimate diverges as $E \rightarrow 0$. However, it is clear that the divergence is truncated at $E=0$ since in that case the mean square distance travelled by the polymer in any direction as a function of time is given simply by

$$
\left\langle x^{2}\right\rangle=2 D t
$$

The average time to travel a distance $\sqrt{N}$ is then

$$
\tau=\frac{N}{2 D}=\frac{3}{2} N^{3}
$$

Equation (9) now tells us that one correlation time is, in the worst case $E \rightarrow 0$ limit, equivalent to $3 N^{4}$ Monte Carlo moves. This gives a very steep increase in the CPU time required to perform the simulation as a function of system size. Only with recent advances in parallel computation, as well as sophisticated multispin coding techniques, has it become possible to perform these calculations with sufficient accuracy to reveal the scaling behavior of the model. Using the SP-1 supercomputer at Cornell University, Barkema, Marko and Widom [11] performed a calculation in which they measured the zero-field diffusion coefficient for systems of a variety of lengths up to $N=100$. They found that the numerical data were well fit by the formula

$$
N^{2} D=\frac{1}{3}\left(1+5 N^{-2 / 3}\right)
$$

It was also found that for sufficiently large $N$, the drift velocity $v$ in a static field of strength $E$ was a function only of the scaling variable $N E$. The drift velocity was obtained for various values of $N$ and $N E$ up to $N=400$ and $N E=20$. In the limit $N \rightarrow \infty$ and $E \rightarrow 0$ it was found that the drift velocity was well fit by:

$$
N^{2} v=N E\left[\left(\frac{1}{3}\right)^{2}+\left(\frac{2 N E}{5}\right)^{2}\right]^{1 / 2}
$$

for all values of $N E$. Thus, $v$ has a finite, non-zero limit as $N \rightarrow \infty$ at fixed $E$ and this limit is proportional to $E|E|$. This result is in agreement with the conclusions of Duke, Semenov and Viovy [14] for a different but related model. 


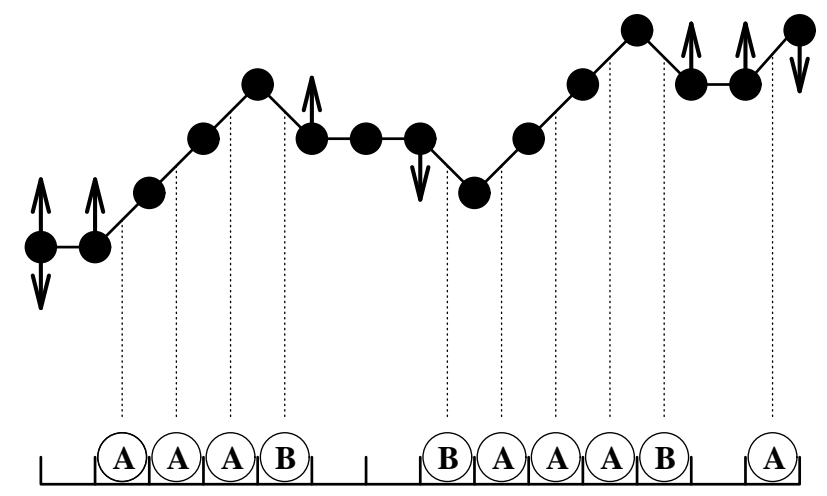

Fig. 2. An illustration of the link between a configuration of the projected repton model, and a configuration in the model with two species of particles.

\section{The repton model as a particle model}

Another representation of the repton model takes the form of a number of particles with hard-sphere repulsion moving around on a one-dimensional lattice. In this representation each link between adjacent reptons in our chain corresponds to a site on the new lattice, and we populate these sites with two species of particles, which we will call types A and B, corresponding to links which slope one way or the other in the projected repton model. For pairs of adjacent reptons which have the same $x$-coordinate we leave the corresponding site on the new lattice empty. This mapping is illustrated in Figure 2 for one particular configuration of the polymer. The rules for the repton model are easily expressed in the language of these particles:

(1) In the interior of the chain the particles are conserved: they can move from site to site on the lattice but they are not created or annihilated.

(2) A particle can only hop to a neighbouring site if that site is not already occupied by another particle. $\square$

(3) A particle on one of the two end sites of the chain can leave the chain - it simply vanishes from the lattice.

(4) If the end site of the chain is empty, a particle of either type can appear there.

In the absence of an electric field, the rates for all particle moves are equal. On average, each particle on the chain tries to hop both to the left and to the right once per unit of simulated time, particles of type A try to enter the

1 The particles are a bit like Fermions in this respect. Notice however that if they were true Fermions, particles of type A and type B would be able to coexist on one lattice site, because they are distinguishable. Since our model is also a completely classical one, it is probably better simply to think of the particles as having hardsphere interactions. 
chain at both the left and right ends once per unit of time, and so do particles of type B. If an electric field is introduced, type A particles will hop to the left at the rate of $\exp (E / 2)$ moves per unit time and to the right at the rate of $\exp (-E / 2)$. Conversely, type $\mathrm{B}$ particles will have rates of $\exp (-E / 2)$ and $\exp (E / 2)$ moves per unit time for hopping to the left and right. Thus, an electric field draws A-particles to the left, and B-particles to the right.

This new physical picture of the repton model is useful, since some things which are not obvious in our other pictures are obvious with this one (and vice versa). For example, given that the electric field will push type A particles one way and type $\mathrm{B}$ ones the other way, it is intuitively reasonable if we start off with a mixture of the two types of particles, that after a while one or more traffic jams are going to build up on the chain, since the two particle types are trying to go in opposite directions but cannot pass one another. Furthermore, these traffic jams will be unstable to fluctuations. If one side of the jam becomes larger than the other - if there are more particles of type A for example than there are of type B - then the larger half will 'push' the smaller half back, and, ultimately, off the end of the chain. When this happens, we end up with a configuration of the chain dominated by one type of particle or the other. What does this situation correspond to if we project it back onto the original repton model? It turns out that it corresponds to a polymer that has got itself lined up along the electric field, so that the reptation motion along the line of the molecule is in exactly the direction the field is trying to push it. This is a situation that is frequently observed in electrophoresis experiments.

Movement of the entire chain along the $x$-axis corresponds to the propagation of particles all the way across the lattice from one end of the system to the other. For example, the centre of mass of the chain will move one step in the positive $x$ direction every time a particle of type A enters the system at the left-hand end and leaves it at the right. It will do the same if a particle of type B moves from right to left. Particles of these types going in the opposite directions will move the chain one lattice spacing in the negative $x$ direction.

Let us denote by $n_{A \rightarrow}$ and $n_{B \rightarrow}$ the number of particles of types $\mathrm{A}$ and $\mathrm{B}$ which cross the system from left to right in a certain time, and by $n_{A \leftarrow}$ and $n_{B \leftarrow}$ the number which go the other way. In the long-time limit the displacement $\Delta x$ of the chain is then given by

$$
\Delta x=\left(n_{A \rightarrow}+n_{B \leftarrow}\right)-\left(n_{A \leftarrow}+n_{B \rightarrow}\right) .
$$

In the $E=0$ case, the two types of particles diffuse in exactly the same fashion, and are therefore indistinguishable, a fact which we can exploit to improve the accuracy of our simulations as follows. If we perform a simulation of the particle model with $E=0$, the movements of the particles will be the same regardless of their type, which means that there is no need to know 


\begin{tabular}{cc}
$N$ & $D N^{2}-\frac{1}{3}$ \\
\hline \hline 20 & $0.2123 \pm 0.0027$ \\
30 & $0.1622 \pm 0.0025$ \\
50 & $0.1079 \pm 0.0023$ \\
70 & $0.0812 \pm 0.0044$ \\
100 & $0.0615 \pm 0.0065$ \\
150 & $0.0332 \pm 0.0077$ \\
200 & $0.0205 \pm 0.0070$ \\
\hline \hline
\end{tabular}

Table 1

The quantity $D N^{2}-\frac{1}{3}$ measures the deviation of the diffusion constant $D$ from its infinite $N$ limit of $1 /\left(3 N^{2}\right)[13]$.

what types they actually are. All we need to do is keep track of the number of particles crossing the system in each direction during the course of the run and then we can assign types A and B to these afterwards in any fashion we choose. In fact, the best statistics are to be obtained by considering every possible such assignment, and averaging over them all. The mean square displacement of the system $\left\langle(\Delta x)^{2}\right\rangle$ is then given by

$$
\begin{aligned}
\left\langle(\Delta x)^{2}\right\rangle & =\left\langle\left[\left(n_{A \rightarrow}-n_{B \rightarrow}\right)-\left(n_{A \leftarrow}-n_{B \leftarrow}\right)\right]^{2}\right\rangle \\
& =\left\langle\left(n_{A \rightarrow}-n_{B \rightarrow}\right)^{2}\right\rangle+\left\langle\left(n_{A \leftarrow}-n_{B \leftarrow}\right)^{2}\right\rangle \\
& =\left(n_{A \rightarrow}+n_{B \rightarrow}\right)+\left(n_{A \leftarrow}+n_{B \leftarrow}\right) \\
& =n_{\rightarrow}+n_{\leftarrow} .
\end{aligned}
$$

The second line here follows since the assignment of As and Bs is uncorrelated with the directions in which the particles cross the system and the third follows from properties of random walks in one dimension. Thus, all we have to do to obtain the diffusion coefficient in a simulation is count the total numbers of particles $n_{\rightarrow}$ and $n_{\leftarrow}$ which cross the lattice in either direction. The implicit average over particle types in (16) gives us an improvement in the efficiency of the calculation of at least an order of magnitude for the typical system sizes we are studying here. We give some preliminary results obtained using this method in Table 1 and Figure 3.

\subsection{Particle densities}

In order to calculate the numbers $n_{\rightarrow}$ and $n_{\leftarrow}$ appearing in Equation (16), we need to keep a record for each particle of whether it entered from the left or the 


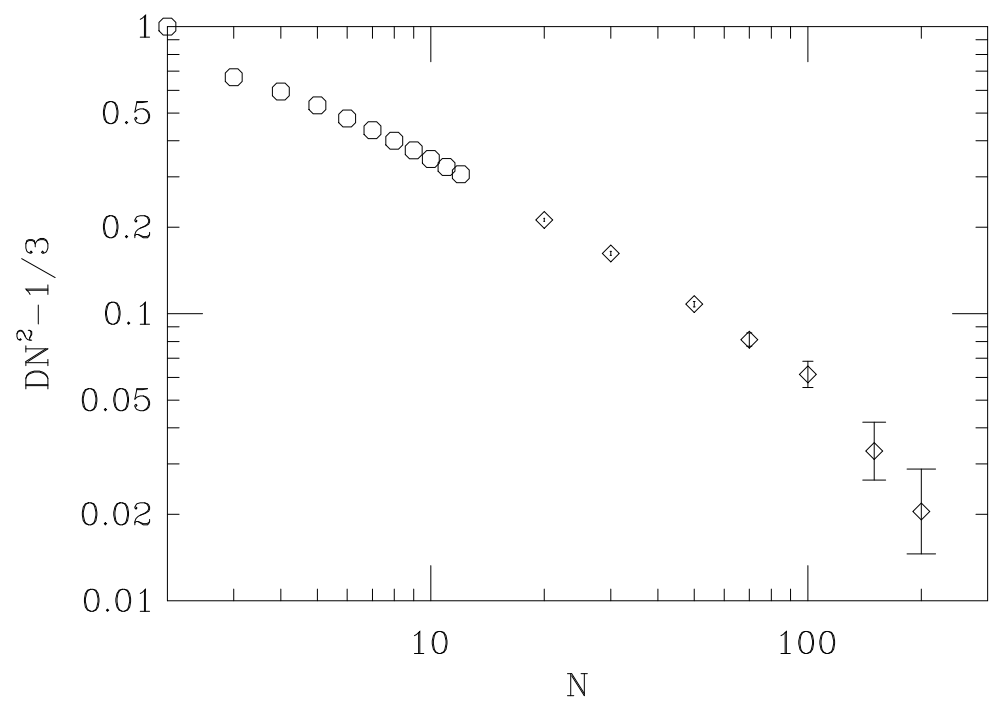

Fig. 3. Log-log plot of $D N^{2}-1 / 3$ as a function of $N$. In the range $20<N \leq 100$, the data is well described by $D N^{2}=1 / 3+5 / 3 N^{-2 / 3}$, as was reported by Barkema, Marko and Widom[11]. The new data for $N \geq 100$ suggests that maybe for larger $N$ this fit is not appropriate any more.

right end of the lattice. Let us label each particle with an $\mathrm{L}$ or an $\mathrm{R}$, depending on the end at which it entered. It turns out that the average densities of L-type and R-type particles possess some interesting features, suggesting a possible route to an exact solution of the repton model.

The dynamics of the $\mathrm{L}$ and $\mathrm{R}$ particles is very similar to that of the $\mathrm{A}$ and $\mathrm{B}$ particles we considered in the last section (they exclude each other, they try to hop left and right with rate 1 , they disappear from either end with rate 1 ), except that $L$ particles enter only from the left, and R particles from the right, both with rate 2 (whereas $\mathrm{A}$ and $\mathrm{B}$ particles entered from either side with rate 1). These rules are depicted in Figure 4.

The total particle density at any site is still equal to $\frac{2}{3}$, just as before. However, since $\mathrm{L}$ particles do not enter at the right end, $\mathrm{R}$ particles do not enter at the left, and the two types are unable to pass one another, the system divides into two domains, each containing just one type of particle as well as vacancies, separated in the middle by an interface. The position of this interface can fluctuate and at times may move all the way to one end of the lattice, allowing particles of one type or the other to pass through the system causing a shift in the centre of mass of the repton chain. However, it is clear that on average the density of particles of type $\mathrm{L}$ will be greater at the left-hand end of the system and vice versa for particles of type R. Because of symmetry between $\mathrm{L}$ and $\mathrm{R}$ particles, the average density of $\mathrm{R}$ particles at a site $i$ is equal to the 

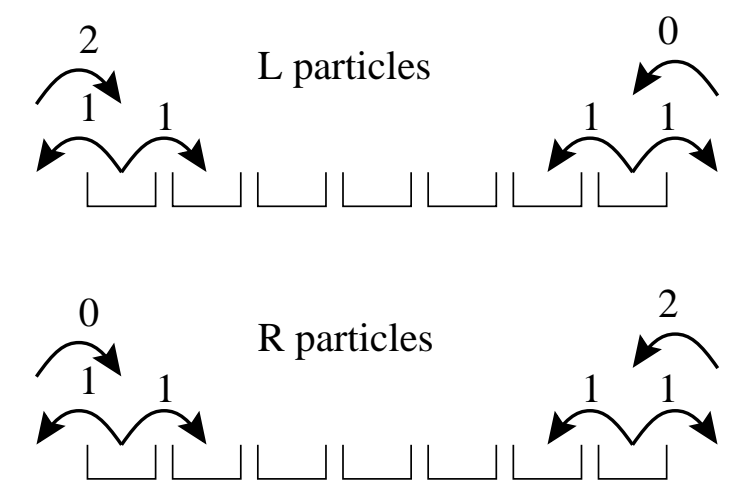

Fig. 4. Illustraction of the dynamics of the $\mathrm{L}$ and $\mathrm{R}$ particles described in the text.

density of $\mathrm{L}$ particles at site $N-i$ :

$$
\rho_{R}(i)=\rho_{L}(N-i) \equiv \rho(i)
$$

We have measured the density profile $\rho(i)$ using our multispin Monte Carlo method for systems of size $N=20,50,100$ and 200. For each value of $N$ we have performed three runs, equivalent, through the magic of multispin coding, to 192 individual simulations, since we performed the runs on a 64-bit computer. The runs were done over $5 \times 10^{8}$ moves for $N=20$ and 50 , and $5 \times 10^{9}$ moves for $N=100$ and 200 . The simulations were started with random configurations with an average particle density of $\frac{2}{3}$ (the expected equilibrium value) and random choices for the interface position. To ensure thermalization, we initially ran the simulations for an additional $10 \%$ of the total number of moves and discarded the data.

In Figures 5 and 6 we have plotted $\rho(i)$ as a function of $i$ on linear and logarithmic scales. In the latter, we observe power-law scaling of $\rho(i)$ as a function of $i$ for small $i$ :

$$
\rho_{R}(i) \sim i^{\alpha}
$$

with an exponent which is independent of the length of the chain:

$$
\alpha=2.70 \pm 0.04 \text {. }
$$

We conjecture that the actual value of this exponent may be $N^{-8 / 3}$ and that it may be related to the sub-dominant scaling of the diffusion coefficient observed by Barkema, Marko and Widom [11].

It is in fact possible to compute the value of the diffusion coefficient from a knowledge of the density profile: the rate with which $\mathrm{L}$ particles ( $\mathrm{R}$ particles) 


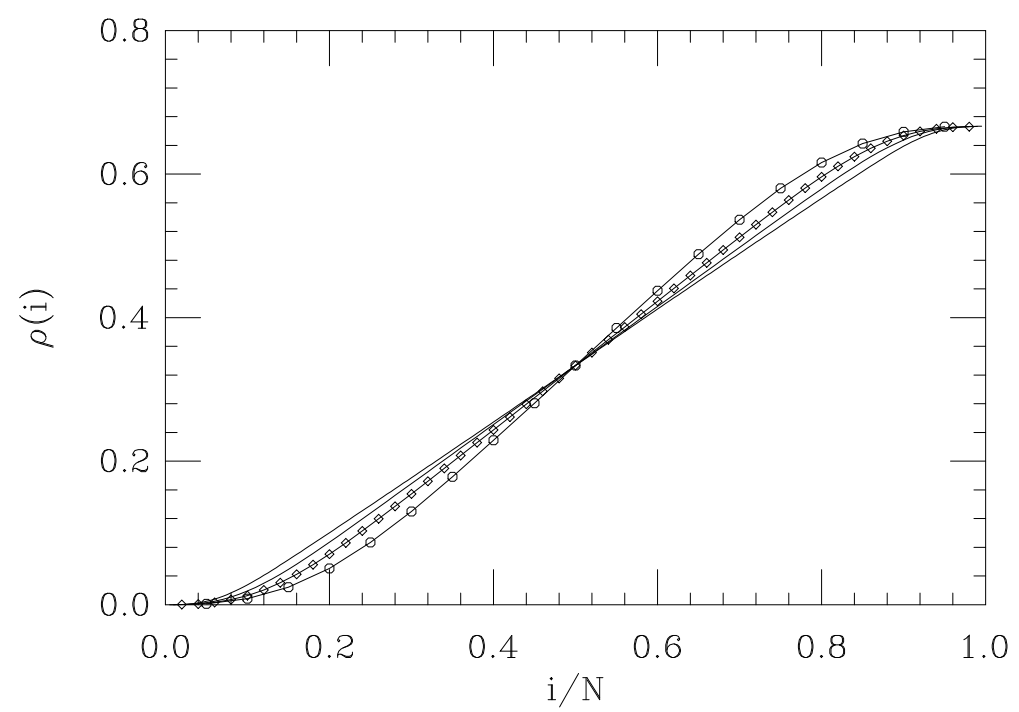

Fig. 5. Density of $\mathrm{R}$ particles at site $i$, as a function of distance along the chain (as a fraction of the total chain length.

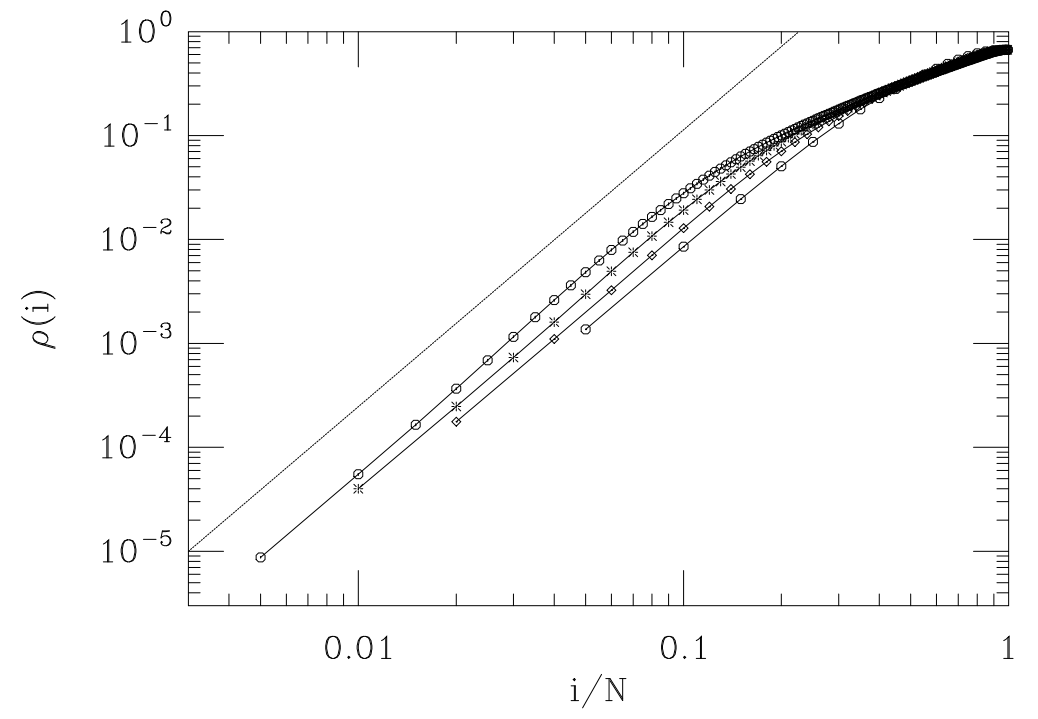

Fig. 6. Log-log plot of the density of $\mathrm{R}$ particles at site $i$, as a function of distance along the chain. For low values of $i$, the density appears to scale as a power-law. The dashed line is added a guide to the eye; it is a power-law with exponent $\frac{8}{3}$.

leave the system at the right-hand (left-hand) end, is strictly equal to their density at the last site. Using Equation (16), we thus obtain:

$$
D=\frac{1}{2} \frac{\partial}{\partial t}\left\langle(\Delta x)^{2}\right\rangle=\rho(1)
$$


This formalism suggests a possible route to an exact solution of the repton model. For a class of models known as asymmetric exclusion models, Derrida et al. [15] have derived an analytic expression for the particle density

profile for any $N$. The dynamics of these models is closely related to that of the particle version of the repton model presented here, and it is possible that the result might be extended to the repton model as well. If so, Equation (20) would then tell us the exact value of the diffusion constant for any $N$.

\section{Conclusions}

We have described the repton model of the agarose gel electrophoresis of DNA, as well as its projection onto a one-dimensional reptation model and a onedimensional particle model. We have reviewed exact analytic results on the model for short chain lengths and numerical studies of longer ones. In addition we have described a new algorithm for simulating the model using the particle representation and presented preliminary results from simulations using this algorithm. In closing we have pointed out that the diffusion constant of the polymer is related in a simple way to the density profile of the particles which suggests a possible route to an exact solution of the model.

\section{Acknowledgements}

One of us (GTB) would like to thank the Santa Fe Institute for their hospitality whilst this work was carried out. This research was funded in part by the DOE under grant number DE-FG02-9OER40542 and by the Santa Fe Institute and DARPA under grant number ONR N00014-95-1-0975.

\section{References}

[1] P. G. de Gennes, J. Chem. Phys. 55, 572 (1971).

[2] M. Rubinstein, Phys. Rev. Lett. 59, 1946 (1987).

[3] T. A. J. Duke, Phys. Rev. Lett. 62, 2877 (1989).

[4] T. A. J. Duke, J. Chem. Phys. 93, 9049 (1990).

[5] T. A. J. Duke, J. Chem. Phys. 93, 9055 (1990).

[6] J. M. J. van Leeuwen and A. Kooiman, Physica A 184, 79 (1992). 
[7] A. Kooiman and J. M. J. van Leeuwen, Physica A 194, 163 (1993).

[8] A. Kooiman and J. M. J. van Leeuwen, J. Chem. Phys. 99, 2247 (1993).

[9] B. Widom, J. L. Viovy and A. D. Defontaines, J. Phys. I France 1, 1759 (1991).

[10] B. Widom, Physica A, in press (1996).

[11] G. T. Barkema, J. F. Marko and B. Widom, Phys. Rev. E 49, 5303 (1994).

[12] G. T. Barkema, C. Caron and J. F. Marko, Biopolymers 38, 665 (1996).

[13] M. Prähofer, Diplomarbeit, Ludwig-Maximilians-Universität München, (1994).

[14] T. A. J. Duke, A. N. Semenov, and J. L. Viovy, Phys. Rev. Lett. 69, 3260 (1992).

[15] B. Derrida, M. R. Evans, V. Hakim, and V. Pasquier, J. Phys. A Math. Gen. 26, 1493 (1993). 